\title{
Ultrasound and Electromyography as Guidance Tools for the Botulinum Toxin Therapy in Cervical Dystonia
}

\author{
G. Salazar*, S. Ferreiro, M. Fragoso, J. Codas, H. Cruz \\ Department of Neurology, Consorci Sanitari de Terrassa CST, Terrassa, Barcelona, Spain \\ Email: *gsalazar@cst.cat
}

How to cite this paper: Salazar, G., Ferreiro, S., Fragoso, M., Codas, J. and Cruz, H. (2021) Ultrasound and Electromyography as Guidance Tools for the Botulinum Toxin Therapy in Cervical Dystonia. Journal of Behavioral and Brain Science, 11, 49-57.

https://doi.org/10.4236/jbbs.2021.112004

Received: January 4, 2021

Accepted: February 19, 2021

Published: February 22, 2021

Copyright $\odot 2021$ by author(s) and Scientific Research Publishing Inc. This work is licensed under the Creative Commons Attribution International License (CC BY 4.0).

http://creativecommons.org/licenses/by/4.0/

\begin{abstract}
Cervical Dystonia (CD) is the most common type of focal dystonia in the movement disorders units of any specialized hospital around the world. Botulinum Toxin (BT) infiltration is the treatment of choice for CD, according to most of the experts around the world, however the efficacy and tolerance of BT therapy in CD depend on the accuracy when BT is released into the muscles. We reviewed the medical literature in regard to the use of guiding tools for the BT infiltration in CD patients. Results: The use of guiding tools such as Ultrasound or EMG definitely improves the accuracy for releasing the BT into the muscles involved according to some authors. Conclusion: the use of Ultrasound and EMG improves the efficacy and reduce the adverse effects in the BT therapy in $\mathrm{CD}$ patients.
\end{abstract}

\section{Keywords}

Botulinum Toxin, Cervical Dystonia, Ultrasound, EMG, Guiding Tools

\section{Introduction}

Cervical Dystonia (CD) is the most common type of dystonia attended in the movement disorders units around the world [1] [2]. Botulinum Toxin (BT) infiltrations have shown to improve the abnormal posture and also reduce the pain related to the $\mathrm{CD}$ [3] [4] [5]. The efficacy and the adverse effects of the BT therapy in $\mathrm{CD}$ depend on the accuracy when the $\mathrm{BT}$ is released in the muscles involved in the abnormal movements [6] [7] [8] [9]. According to some authors, the efficacy of BT infiltration also depends on the use of guiding tools such as Ultrasound, EMG or electrical stimulation in order to improve the accuracy in the muscles involved [9] [10] [11] [12]; Nevertheless, there is few infor- 
mation published in the medical literature in regard to this issue. We review in this article some of these techniques used nowadays as guiding tools in the BT infiltration in $\mathrm{CD}$ patients and describe the advantages and disadvantages of all of them.

\section{The Concepts of Caput and Collis and How These Concepts Suggest the Use of Any Guiding Tool in the Botulinum Toxin Infiltrations for Cervical Dystonia Patients}

The common classification of $\mathrm{CD}$, according to the phenomenology of the abnormal movements, states four types of CD (Torticollis, Laterocollis, Anterocollis, Retrocollis) [13]. However, this CD classification could be inadequate according to more recent publications of the same authors [14] [15] [16]. When the concepts of Caput and Collis were introduced in the neck muscles phenomenology of cervical dystonia, the understanding of the muscles involved in the abnormal postures in $\mathrm{CD}$ patients clearly changed for the majority of authors. Despite the fact, the direction of the head is similar in both conditions, the muscles involved are partially different and these differences can determine the muscles targets to be infiltrated changing the efficacy and the adverse effects depending on the muscles target chosen.

The concept of Caput is referred to the movement of the head with few or not neck compromise, whereas the concept of Collis is referred to both, neck and head movement simultaneously, for this reason, the muscles involved in Caput and Collis are partially different and the rationale before deciding what muscles targets must be infiltrated change depending on Caput or Collis dystonic posture.

In regards to this classification, we have to consider the alignment of some anatomical points of reference such as the nose, the chin, the larynx and the cervical/thoracic spine angle, therefore, we can observe 4 possibilities in the caput sub-group: (Torticaput, Laterocaput, Anterocaput y Retrocaput); Torticaput (head rotation) implies the non-alignment of the nose with the middle of the larynx, observing the larynx at the middle of the neck whereas the nose is observed in another lateral plane towards the side where the head is rotated, in the case of Laterocaput (lateral head deviation) which implies also the non-nose and larynx alignment observing the larynx at the middle of the neck whereas the nose is observed in a lateral side where the head is lateralized. In the case Anterocaput/Retrocaput there is no angle in the cervical and thoracic spine line as you can observe in the picture (Figure 1).

On the other hand, in the Collis sub-group, we can also observe 4 other possibilities, the first one: Torticollis which is the head and neck rotation with the alignment of the nose and larynx; Laterocollis: the head and neck lateral deviation with the nose and larynx alignment; in the cases of Retrol Anterocollis there is an angle in the cervical and thoracic spine line, look at the Figures (Figure 1). 


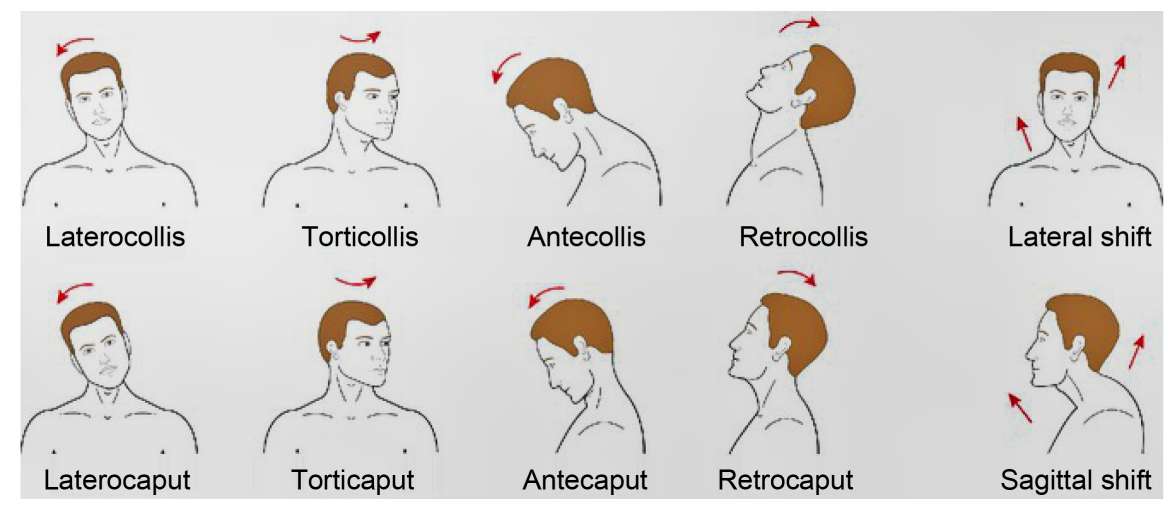

Figure 1. Cervical dystonia classification: caput and collis concepts.

$\mathrm{CD}$ patients can also show more complex abnormal postures, $60 \%$ of $\mathrm{CD}$ patients show only Collis or Caput isolated dystonic postures, however the other $40 \%$ of patients show combination of both, Caput and Collis or many other combinations of the aforementioned abnormal cervical postures. Having said that, it is easy to understand that the experts around the world have been using different guiding methods in the past two decades, in order to achieve adequately the muscles involved in the $\mathrm{CD}$ in order to release accurately the $\mathrm{BT}$ in the appropriate muscles involved in the dystonic posture, improving the efficacy and avoiding the adverse effects associated to the inadequate BT placement in cervical dystonia patients.

\section{Guidance for Botulinum Toxin Infiltration in Cervical Dystonia Patients. Why Should We Use One of Them?}

Manual Needle Placement (MNP) has been traditionally the most commonly used guiding method to infiltrate BT in CD patients [17] [18]. This technique consists in the muscle palpation, recognizing the possible muscles involved in the phenomenology of the dystonic posture, also taking into account some anatomical landmarks to identify the muscles targets ensuring the BT is released in the adequate muscle; nevertheless, according to some authors, this technique requires a deep knowledge of the neck anatomy and it's logically affected for the physical characteristics of every patient, therefore, the accuracy and the adverse effects change substantially depend on the experience of the evaluator and due to the patient's neck particular anatomy. Probably this technique has the advantage of saving time in the procedure, however there is an enormous limitation to identify adequately the muscles targets in the case of complex CD and even more when patients show short and fatty necks representing a challenge for the physician to infiltrate BT accurately and safety.

Other guiding methods such as Electrical Stimulation (ES), Electromyography (EMG) and Ultrasound (US) have also been reported successful for BT infiltration in spastic patients as well as in $\mathrm{CD}$ patients according to some authors [19] [20] [21] [22].

The efficacy of BT infiltrations in CD has been reported from $60 \%$ to $70 \%$ de- 
pending on the series published [8] [9] and the adverse effects associated to BT infiltrations such as dysphagia, neck weakness and pain in the infiltration points are also commonly reported and such adverse effects have been traditionally related to more complex cervical dystonia sub-types [10] [11], because in such cases a bigger variety of muscles are simultaneously involved in the abnormal posture, therefore, the evaluator frequently faces serious difficulties to decide what muscles must be infiltrated and also avoiding other anatomical structures (nerves and vessels) then after ameliorating the side effects. The spreading of BT to other undesirable muscles (pharyngeals), near to the lateral cervical muscles (sternocleidomastoids and scalenus), is the cause of dysphagia in most of the CD patients; on the other hand, the cervical weakness after BT infiltration has also been related to the spreading of BT to deeper neck flexors muscles mainly in the posterior cervical region such as in multifidus muscles group. As we can see, the lack of accuracy of the BT infiltration is the most important point to take into account in regard to the efficacy and adverse effects in CD dystonia patients after BT infiltration as aforementioned, therefore there is a consensus between the authors, in the use of any kind of guiding tool, in the BT infiltration of CD dystonia patients in which the physician feels comfortable and familiarized.

Nowadays, most of the authors propose the use of EMG or US as guiding tools to infiltrate BT in CD patients; Electrical stimulation is still used for some groups in patients with spasticity in upper and lower limbs however it is not used in cervical dystonia. We will specifically describe the useful of EMG and US in CD patients as the most common techniques used as guidance tools in the BT therapy in CP patients

\section{EMG as Guidance in the Botulinum Toxin Infiltration in Cervical Dystonia}

Studies using electromyographic needle in the evaluation of CD patients have revealed that clinical examination alone is insufficient for determining which muscles contribute to the dystonic movement [21]. When compared to needle Electromyography (EMG), movement disorders specialists correctly identified only 59\% of active muscles. The selection of incorrect muscles for injection of Botulinum toxin may explain why some patients have a sub-optimal response and show adverse effects [21] [22].

The significance of electromyography guidance in BT treatment has been much debated for so many years, however some authors have shown that patients treated guided by EMG, clinical outcome, was better than in patients treated based on clinical judgement alone $(\mathrm{p}=0.05)$, concluding that treatment with BT guided by EMG results in better clinical outcome than treatment without EMG and reduces the amount of BT used [21]. On the other hand, it is well-known that EMG activity commonly changes and is reduced as a result of the BT activity on the motor ending and the reduction of the EMG overactivity usually represents a problem for future infiltrations because the overactivity helps us to decide the 
best place where botulinum toxin should be infiltrated, therefore it represents a limitation for the EMG as a guidance in patients who need continuous botulinum toxin infiltrations [22]. As long as EMG can guide us to infiltrate the most overactive muscles, it cannot help us to avoid important anatomical cervical structures such as blood vessels (vertebral artery, carotid artery or jugular veins) or common nerves such as cervical plexus or vagal nerve or C2 nerves when upper cervical muscles are involved in the abnormal posture. Therefore, it's impossible to visualize this anatomical structure and there is a high risk of injure them representing another important limitation of EMG as a guiding tool for botulinum toxin infiltration mainly in complex cervical dystonia [23] [24].

\section{Ultrasound as a Guidance Tool in Cervical Dystonia}

Ultrasonography (US) allows non-invasive, real-time imaging of muscular and glandular tissues and their surrounding structures. It can visualize, guide, and standardize the entire procedure of Botulinum toxin application. Small, randomized studies suggest that US-guidance can improve therapeutic efficacy and reduce adverse effects of Botulinum therapy when compared to conventional placement [8] [9] [10] [19] [20]. US-guidance should be used in forearm muscles when functionality is important, and in selected cervical muscles in complex cervical dystonia.

In regard to ultrasound as a guidance to infiltrate muscles in cervical dystonia, we want to mention three important aspects to take into account when the muscles targets are analyzed:

1) The phenology of the dystonic movements; 2) The muscle hypertrophy and 3) The echo-hyperintensity of the muscles involved in the dystonia.

In regard to the phenomenology, US allows us to identify the muscles which previously have been clinically identified and related to the abnormal postures (Torticaput, Torticillis, Lateraocaput, Laterocollis or some of them in combination) [8] [9] [10] [15] [16]. Concomitantly, the muscle hypertrophy points out the most overactive muscles involved in the abnormal posture, whereas the muscle hypertrophy is greater the muscle overactivity is also greater; we determine the thickness of the muscle and compare it with the same contralateral muscle in order to determine the muscle trophism (Ex. the right splenium with the left splenium muscle). Finally, With US, enhanced echo intensity (EI) represents changes caused by increased intramuscular fibrous and adipose tissue for multiple causes already reported for some authors [25] [26] [27] and the muscle echo-hyperintensity also has been related to the hyperactivity that a muscle could have been experienced in a dystonic posture. These three points could be helpful when we analyze the neck muscles to infiltrate BT in cervical dystonia patients using US as a guidance tool. In regard to the adverse effects, US as a real time imaging technic visualizes nerves and vessels as we mentioned before avoiding important injuries on anatomical structures such as the cervical plexus or the jugular vein; as a disadvantage, the muscles functionality is not well-evaluated as 
it can be done with EMG, nevertheless some indirect signs such as muscle hypertrophy and the muscle echo-hyperintensity can be helpful to adequately identify the most hyperactive muscles [28] [29].

\section{Discussion}

Cervical dystonia patients are commonly attended in specialized movement disorders units around the world [14] [17], those patients frequently represent a therapeutical challenge because the complexity of the muscles involved in the abnormal posture and the anatomical difficulties in many cases of $\mathrm{CD}$. Botulinum toxin infiltrations have shown to be effective to reduce the pain and the abnormal postures on these patients (5). However, the adverse effects and the efficacy of BT infiltrations have been reported with important differences depending on the series published in the medical literature. The Guidance tools for $\mathrm{BT}$ infiltrations in CD patients are not always used in some movements disorders units giving preference to manual needle placement, despite the better results showed with the use of guiding tools such as EMG and US [9] [17]-[22]. The accuracy of BT infiltrations clearly decreases when the guidance tools are not used in the BT therapy for CD patients therefore the efficacy decreases and the adverse effects such as dysphagia and cervical weakness clearly increase in most of the series published in the medical literature [8] [9] [20].

In the case of EMG, the efficacy is directly related to the overactivity of the muscle ending plate, giving excellent results in $\mathrm{CD}$ patients who also show spasms and tremor among the dystonic posture. However, this electric overactivity is progressively reduced due to repetitive BT infiltrations, furthermore the EMG usefulness is limited in the case of complex cervical dystonia patients where multiple and deeper cervical muscles are involved because the complexity of the abnormal posture represents a high risk of injuring noble structures such as cervical vessels and nerves is a real.

Ultrasound as a guiding tool for Botulinum toxin therapy has been used widely in the last 10 years, mainly in spastic patients improving results in efficacy and tolerance with an important level of scientific evidence. In CD patients, US allows an exact release of BT in the muscles really involved in the abnormal postures avoiding important anatomical structures and adverse effects among better results which have been reported for many experts dedicated to movement disorders [9] [17] [18] [20] [21] [23]. However, randomized, cross over, parallel control studies are still needed meanwhile the US devices are technically improved every year, nevertheless this technical improvement must have an impact in an easier training for physicians in order to understand and handle better the US devices and improving the efficacy and tolerance of BT therapy in CD patients.

\section{Conclusion}

BT infiltrations for CD patients are performed with or without guidance tools depending on the experience of specialist dedicated to movement disorders. 
Manual Needle placement with muscle localization (no guidance) is probably the most common method still performed for BT therapy elsewhere, however other guidance methods such as EMG and US have shown more accuracy and less adverse effects and should be performed to improve the results and reduce adverse effects according to the majority of experts around the world; the choice of the guidance tool depends on the technique in which the physician is familiarized and feels comfortable, however the new developments of software and better images point out the ultrasound as a preferent choice for the new generations of physicians involved in the BT treatment for $\mathrm{CD}$ patients.

\section{Conflicts of Interest}

The authors declare no conflicts of interest regarding the publication of this paper.

\section{References}

[1] Defazio, G., Jankovic, J., Giel, J.L. and Papapetropoulos, S. (2013) Descriptive Epidemiology of Cervical Dystonia. Tremor and Other Hyperkinetic Movements, 3 , tre-03-1993. https://doi.org/10.5334/tohm.170

[2] Jankovic, J., Tsui, J. and Bergeron, C. (2007) Prevalence of Cervical Dystonia and Spasmodic Torticollis in the United States General Population. Parkinsonism \& Related Disorders, 13, 411-416. https://doi.org/10.1016/j.parkreldis.2007.02.005

[3] Jankovic, J. and Schwartz, K. (1990) Botulinum Toxin Injections for Cervical Dystonia. Neurology, 40, 277-280. https://doi.org/10.1212/WNL.40.2.277

[4] Jankovic, J. (2006) Botulinum Toxin Therapy for Cervical Dystonia. Neurotoxicity Research, 9, 145-148. https://doi.org/10.1007/BF03033933

[5] Hefter, H., Benecke, R., Erbguth, F., Jost, W., Reichel, G. and Wissel, J. (2013) An Open-Label Cohort Study of the Improvement of Quality of Life and Pain in de Novo Cervical Dystonia Patients after Injections with 500 U Botulinum Toxin A (Dysport). BMJ Open, 3, e001853. https://doi.org/10.1136/bmjopen-2012-001853

[6] Bhidayasiri, R. (2011) Treatment of Complex Cervical Dystonia with Botulinum Toxin: Involvement of Deep-Cervical Muscles May Contribute to Suboptimal Responses. Parkinsonism \& Related Disorders, 17, S20-S24. https://doi.org/10.1016/j.parkreldis.2011.06.015

[7] Reichel, G. (2014) Examination and Treatment of Complex Cervical Dystonia. In: Manual of Botulinum Toxin Therapy, Cambridge University Press, Cambridge, 35-45. https://doi.org/10.1017/CBO9781139178068.007

[8] Picelli, A., Roncari, L., Baldessarelli, S., Berto, G., Lobba, D., Santamato, A., Fiore, P. and Smania, N.J. (2014) Accuracy of Botulinum Toxin Type A Injection into the Forearm Muscles of Chronic Stroke Patients with Spastic Flexed Wrist and Clenched Fist: Manual Needle Placement Evaluated Using Ultrasonography. Journal of Rehabilitation Medicine, 46, 1042-1045. https://doi.org/10.2340/16501977-1871

[9] Ko, Y.D., Yun, S.I., Ryoo, D., Chung, M.E. and Park, J. (2020) Accuracy of Ultrasound-Guided and Non-Guided Botulinum Toxin Injection into Neck Muscles Involved in Cervical Dystonia: A Cadaveric Study. Annals of Rehabilitation Medicine, 44, 370-377.

[10] Picelli, A., Bonetti, P., Fontana, C., Barausse, M., Dambruoso, F., Gajofatto, F., et al. (2012) Accuracy of Botulinum Toxin Type A Injection into the Gastrocnemius Muscle 
of Adults with Spastic Equinus: Manual Needle Placement and Electrical Stimulation Guidance Compared Using Ultrasonography. Journal of Rehabilitation Medicine, 44, 450-452. https://doi.org/10.2340/16501977-0970

[11] Lim, E.C., Quek, A.M. and Seet, R.C. (2011) Accurate Targeting of Botulinum Toxin Injections: How to and Why. Parkinsonism \& Related Disorders, 17, S34-S39. https://doi.org/10.1016/j.parkreldis.2011.06.016

[12] Poewe, W. and Wissel, J. (1993) Use of Botulinum Toxin in the Treatment of Cervical Dystonia. Bailliere's Clinical Neurology, 2, 179-185.

[13] Reichel, G., Stenner, A. and Jahn, A. (2009) The Phenomenology of Cervical Dystonia. Fortschritte der Neurologie-Psychiatrie, 77, 272-277.

https://doi.org/10.1055/s-0028-1109416

[14] Jost, W.H. and Tatu, L. (2015) Selection of Muscles for Botulinum Toxin Injections in Cervical Dystonia. Movement Disorders Clinical Practice, 2, 224-226. https://doi.org/10.1002/mdc3.12172

[15] Stenner, A. and Reiche, G. (2015) A New Classification of Cervical Dystonia for Botulinum Toxin Therapy: The Col Cap Concept. Movement Disorders, 357, e288. https://doi.org/10.1016/j.jns.2015.08.1006

[16] Tsui, J.K. (1995) Cervical Dystonia. In: Tsui, J.K. and Calne, D., Eds., Handbook of Dystonia, Marcel Dekker, Inc., New York, 115-127.

[17] Schnitzler, A., Roche, N., Denormandie, P., Lautridou, C., Parratte, B. and Genet, F. (2012) Manual Needle Placement: Accuracy of Botulinum Toxin A Injections. Muscle \& Nerve, 46, 531-534. https://doi.org/10.1002/mus.23410

[18] Santamato, A., Micello, M.F., Panza, F., Fortunato, F., Baricich, A., Cisari, C., Pilotto, A., Logroscino, G., Fiore, P. and Ranieri, M. (2014) Can Botulinum Toxin Type A Injection Technique Influence the Clinical Outcome of Patients with Post-Stroke Upper Limb Spasticity? A Randomized Controlled Trial Comparing Manual Needle Placement and Ultrasound-Guided Injection Techniques. Journal of the Neurological Sciences, 347, 39-43. https://doi.org/10.1016/j.jns.2014.09.016

[19] Walter, U. and Dressler, D. (2014) Ultrasound-Guided Botulinum Toxin Injections in Neurology: Technique, Indications and Future Perspectives. Expert Review of Neurotherapeutics, 14, 923-936. https://doi.org/10.1586/14737175.2014.936387

[20] Hong, J.S., Sathe, G.G., Niyonkuru, C. and Munin, M.C. (2012) Elimination of Dysphagia Using Ultrasound Guidance for Botulinum Toxin Injections in Cervical Dystonia. Muscle \& Nerve, 46, 535-539. https://doi.org/10.1002/mus.23409

[21] O'Brien, C.F. (1997) Injection Techniques for Botulinum Toxin Using Electromyography and Electrical Stimulation. Muscle and Nerve, 20, S176-S180. https://doi.org/10.1002/(SICI)1097-4598(1997)6+<176::AID-MUS12>3.0.CO;2-4

[22] Lee, L.H., Chang, W.N. and Chang, C.T. (2004) The Finding and Evaluation of EMG-Guided BOTOX Injection in Cervical Dystonia. Acta Neurologica Taiwanica, 13, 71-76.

[23] Werdelin, L., Dalager, T., Fuglsang-Frederiksen, A., Regeur, L., Karlsborg, M., Korbo, L., Munck, O. and Winge, K. (2011) The Utility of EMG Interference Pattern Analysis in Botulinum Toxin Treatment of Torticollis: A Randomised, Controlled and Blinded Study. Clinical Neurophysiology, 122, 2305-2309.

https://doi.org/10.1016/j.clinph.2011.04.012

[24] Gelb, D.J., Yoshimura, D.M., Olney, R.K., Lowenstein, D.H. and Aminoff, M.J. (1991) Change in Pattern of Muscle Activity Following Botulinum Toxin Injections for Torticollis. Annals of Neurology, 29, 370-376.

https://doi.org/10.1002/ana.410290407 
[25] Reimers, K., Reimers, C.D., Wagner, S., Paetzke, I. and Pongratz, D.E. (1993) Skeletal Muscle Sonography: A Correlative Study of Echogenicity and Morphology. Journal of Ultrasound in Medicine, 12, 73-77. https://doi.org/10.7863/jum.1993.12.2.73

[26] Pillen, S., Tak, R.O., Zwarts, M.J., et al. (2009) Skeletal Muscle Ultrasound: Correlation between Fibrous Tissue and Echo Intensity. Ultrasound in Medicine and Biology, 35, 443-446. https://doi.org/10.1016/j.ultrasmedbio.2008.09.016

[27] Reimers, C.D., Schlotter, B., Eicke, B.M. and Witt, T.N. (1996) Calf Enlargement in Neuromuscular Diseases: A Quantitative Ultrasound Study in 350 Patients and Review of the Literature. Journal of the Neurological Sciences, 143, 46-56.

https://doi.org/10.1016/S0022-510X(96)00037-8

[28] Franchi, M.V., Longo, S., Mallinson, J., Quinlan, J.I., Taylor, T., Greenhaff, P.L. and Narici, M.V. (2018) Muscle Thickness Correlates to Muscle Cross-Sectional Area in the Assessment of Strength Training-Induced Hypertrophy. Scandinavian Journal of Medicine \& Science in Sports, 28, 846-853. https://doi.org/10.1111/sms.12961

[29] Watanabe, Y., Yamada, Y., Fukumoto, Y., Ishihara, T., Yokoyama, K., Yoshida, T., Miyake, M., Yamagata, E. and Kimura, M. (2013) Echo Intensity Obtained from Ultrasonography Images Reflecting Muscle Strength in Elderly Men. Clinical Interventions in Aging, 8, 993-998. https://doi.org/10.2147/CIA.S47263 